\title{
CONTROL DE SAPROLEGNIALES POR ACIDO ACETICO, CLORURO DE SODIO Y VERDE MALAQUITA EN HUEVOS DE TRUCHA ARCOIRIS
}

\author{
(Saprolegniales control by acetic acid, sodium chloride and \\ malaquite green in eggs of rainbow trout)
}

Valia Vivar, M. \& Fabian Bernal, L.

Universidad Católica de Valparaíso, Facultad de Recursos Naturales, Escuela de Ciencias del Mar, Casilla 1020, Valparaíso, Chile.

Palabras clave: Control fúngico, Saprolegniales, hucvos de trucha

Key words: Fungal control, Saprolegnales. trout cggg

\section{RESUMEN}

Con la finalidad de determinar la eficacia, como agentes inhibidores del crecimiento fingico de Suprolegniales, en ovas de mucharromis, se procedio a Ia evaluacion de tralamientos con átho acético (100 ppm, 300 ppn y 500 ppm), clowno de sodio (20\%) y verte malaquin (2,5 ppn). Los paramenosmedidos fueron: la mortalidad iolal de los huevos hasta que sistos presentaran el ojo visible y la defomidal de abvines con snco. Los tratamientos se aplicaron whizand ha tecnica de corm te de flujo, efechllada dia por medio y con liempos de 60 mimutos de duración. Este procétimiento abarcó desde la fecundación hasta la aparición de los ojos.

E/ verde malaquila, presentó la menor morfalidad lotal de huevos $(12,95 \%)$, seguido por el acilo acético a 500 ppm (16,96\%). En las ovas murras con hongos alheridos a su superficte se registró un $6,76 \%$ para verte malaquitay un 11,72\% para el ácido acético a 500 ppm. Con el clomm de solio se obumo la maver proporcion en anbos casos. Loshueves ratados con verde malaquita, registraron ma alla incikencia de alevines consaco deformes $(13,5 \%$ ), micamas para los resiantes tratamientos, fucron inferiores al $7 \%$.

Es posible conchir que el cicido acéfico en concentraciones de 500 ppm puede ser athiado como sustitulo al verde malatula para commolar infecoiones micóblcas en ovas, va que no prodnce elevalos niveles de motalidad en los huevos y no malue a la fomacion de alevines con saco deformes:

\section{INTRODUCCION}

El potencial productivo de uma piscicultura está sustcotado por la producción de ovis. En esta clapa, los principales obstáculos son: los problemas provocados

\section{SUMMARY}

In order to asses the efficiency as inhibiting agents of fingal growth of Saprolegniales in raimbow trout eggs, treaments with acet acid (100 ppm, 300ppm, and 500 ppm), solinm chlonide (20) and halaquite green (2,5 ppm) were evaluated. Paranters laken into account were: total mortality of eggs wnil they made their eyes yisible and showed their bags distorled.

Treatments were carried out in allerhative days by using the flow cut off technique for a $60 \mathrm{~min}$ period. This procedure was accomplished since fecundation until the appearance of eyes.

The least total mortality of eggs (12,95\%) was obsened in malaquite green, followed by the acetic acid a 500 ppm (16,96\%). As to dead eggs bearing fungi on their surface, $6,76 \%$ was recorter with malaquite green and $11,72 \%$ wilh acelic acid at 500 ppm. In bolh cases the highes/ dafe occured wh solim chloride.

Eggs treated with malaquile green revealed a high incidence of fry having distorted bags $(13,5 \%)$ whereas the remaining treatments were lesser than $7 \%$.

It is concluded that the aceric acid in 500 ppm concentrations can be used as a substitule to malaquite green in order lo control mycotic infections in troul eggs since it does not couse high levels of mortality in the latter nor induces the formation of fry with distorted bags.

por la alta densidad con la cual se trabaja, la manipulación de los hucvos, las variaciones de temperalura y la presencia de agentes patógenos en el agua (Bailey, 1984). Todo esto hace que los hucvos cn desarrollo sean muy sensibles a la prolircración de infeccioncs micólicas, causadas 
principalmente por hongos del géchcro Suproluzgniat. Scgún Torroclla (1988), cslas infocciones se presentan frecuentcmente en cullivos intensivos de salmónidos y generalmenc son causadas por la combinación de un agente infeccioso y cl cstrés ambicntal. siluatción que provoca la progresión de la cnfermedad y a scces la muerte de los hucios. Es importante senalar que el acuicullor realiza variadas acriviclades que causin estrés en los peces. cutre cllas destacam: la separación por tamaños. cl transporte, la alimentación. los desdobles. las condiciones de desove, la incubación. ctc. (Kitancharoen \& Hatai, 1997).

La susceplibilidad de los hueros de peces a has infecciones micólicas dependen de varios factores. lales como: la alta densidad de cullivo que promucre la infección por hongos( Mendo\%a. 1994). cl Mujo de agua del sistema de incubación que no es sulicicnte para critar la adhesión de las zoosponas a los hucros (Rach et al., 1998) y la calidad del agua. Además. ha presencia de hueros mucrlos y la matcria orgánica disucla en el agua provec un excelcnte sustrato para el ascntimiento y desarrollo de los hongos lo que hace necesaria la adición de agentes antifüngicos (Willonghby \& Roberts. 1992).

El verde malaquila se ha usado por muchos años como agente fungicida para combaltir estc lipo de infección. pero desgraciadamente diversos cstudios han demostado las carncteristicals teratogénicas de este compues10 (Meyer \& Jorgensen, 1984: Noga. 1943. Making et al. 19) : Rach et al, 1997). Por lo anterior investigadorcs como Taylor \& Bailes: (1979): Aldcrman. (1982); Bailcy: (1984): Bailoy \& Jefroy: (1989): Schreck et al., (1990); Edgell etal, (1993): Dawson at a1. (1944): Marking of al., (1994): Fitzpatrick ef at. (1995): Schricr ef al., (19\%6): Rachet al. (1997), ham realizado estudios para proyectar potenciales sustancias antimicóticas que remplacen al verde malaquita.

No obstamle. se hace necesirio seguir imestigando para dectominar las medidas profilicicas a aplicar en las pisciculluras. pama climinar o minimixar las infecciones por hongos. como los procedimientos tendientes a facilitar ce diagnóstico y la aplicación de tcrapias adccuadas y oportumas.

En cl presente cstudio se craluó la actividad anifüngica del ácido ać́ico. cloruro de sodio y verde makquita. ch la mortalidad de ovas y en la deformidad de alevines con saco.

\section{MATERIALES Y METODOS}

La experiencia fue desarrollada en la Piscicullura de Río Blanco. siluada a $1 .+20 \mathrm{~m}$. de allum en la $\%$ ona precordilicrana de la comma de los Andes, V región.
Chile. con comperaturas que flucharon entre 1 y $8^{\circ} \mathrm{C}$.

Sc whilizaron aprovimadamente 15.000 huevos de trucha arcoiris, recićn fecundados, que fueron deposilados en canastillos de incubación con tres divisioncs en su interior $(+5 \times 20 \times 25 \mathrm{~cm})$. ch cada una de las cuales se dispusicron aproximadamente 1000 hucros.

En la cxpericncia se utilizaron 5 canastillos de aluminio, "a los que se les asignó uno de los siguientes trammicntos:

- Camastillo No 1: Verde malaguita (2.5 ppm)

- Camasillo No 2: Cloruro de sodio $(20 \%)$

- Canastillo No 3: Acido acćlico (100 ppiii)

- Canastillo No 4: Acido acético (300 ppm)

- Camastillo No5: Acido acćtico (500 ppm)

Para la incubación de los hucros, los canastillos fueron distribuitos cn balcas horizontales de fibra de vidrio con un volumen útil de $0.1056 \mathrm{~m}^{3}$. Las bateas se alimentaron con un flujo de agua ubicado on forma perpendicular a ellas por tho de sus exiremos permitiendo asi la independencia de los tratamienos on las mismas.

Los tratamicutos se aplicaron cada 36 horas durante un lapso de 36 dias. periodo cn cl cual las ovas presentaron los ojos visibles (ova con ojo). Los tratamientos comenzaron a aplicarse 3 dias despućs de la fecundación. una vee realizado of "picking" (limpicza de huevos muertos). Consisticron en aplicar la solución respectiva en la batca de incubación y contar el fujo del agua para reanudarlo al cabo de una hora.

Scis días después de la aparición del ojo se realizó cl "shocking" (caida desde lm de allo) y posicriormente se procedió a contar y observar los hucros mucr10s. Con ayuda de una lupa csicrcoscópica se deicrminó d porcentaje de ovas mucras infectadas con hifas, según la siguicnte proporción:

$$
\underset{\text { atribuida ahongos }}{\% \text { Hucros con hifas } \times 100}=\frac{\text { Hucros mucros }}{\text { Holidnd }}
$$

Cuando la lolalidad de las ovas sobrevivicntes cclosionaron. se contó los alcrincs con saco que prescnlaban deformacioncs. Para cllo, se lomó uma mucsira alcatoria de 200 alcrines con saco. desde citla una de las divisiones. Sc observó a la hupa la prescncia de individuos deformes. tomando como parameros la curatura de la columna vericbral y mallormacioncs de la mandibula. Se usatron estos parámetros de deformidad debido a que son facilmente distingibles

Considerando que la experiencia fue realizada de acuerdo a un discrio complelamente allentorizalo a un factor. balanceado (Ostlc. 1970). se clectuó un Anailisis de Deviama para un moelclo cxperimenlal a un fictor, compleándose para cllo. cl sofware GUL (Modelos Lineales Generalizados). 


\section{RESULTADOS}

La moralidad lotal hasia la chapa de ova con ojo. fluctuo chtre 12,95 y $29.92 \%$ cn los tratimiculos con rerde malaquila y cloruro de sodio. En las ovas tratadas con ácido acćlico a 500 ppm, sc aprecia tma monalidad media de $16.96 \%$ cn cambio para los tramicnos a 100 ppm y 300 ppm las mortalidades medias obseradas presentan una pequeña diferencia. alcamando valores de $20,49 \%$ : $21.64 \%$ respectivancole. Al hacer el anilisis esladistico. sc pudo detcminar que ninguma de las functones de culace propucstas permilión anuste salisfictorio de los nodelos; a pesar de eslo. pudicron ser usadas para hacer compataciones cmire tratamicntos. El ácido accico a concontaciones de 100 ppm y 300 ppm no presena diferencins significativas on la proporción de mortalidad. lo que hace a ambos manmichlos esholisticamente iguales culresi $\left(D=277.54 ; X^{2}=9,487\right.$ con $\left.\alpha=0.05 \mathrm{yg} .1=4\right)$. Adcmás se pucde decir que cl Inamicnto con verde malaquita fue el más eficay. ya que oburo la menor moralidad total. seguido luego por el matumicnto de ácido actico a 500 ppm. Finalmente d clorturo de sodio. presentó la mayor mortalidad tolal del periodo.

En cuano al porcentaje de ovas muertas que presentabun hifas cn su superficic. se pudo apreciar que los tratamicntos con verde malaquita, deido acclico a 500 ppm y sal comin, sigucn la misma tondencia que la mortalidad total. prescutando valores promedios de $6,76 \% 11,72 \% \mathrm{y}$ $22,+5 \%$ respectivamente. Los Iratamicntos con ácido acélico a 100 ppm y 300 ppm en cmbio, no registran csta Iondencia (Tubla 1 ).

Al igual que en cl análisis cstadistico antes realizato, sc pudo determinar que ninguna de las funciones de cnlace propuestas permitió un ajuste salisfactorio de los modelos. A pesir de cllo, pudieron ser usadas para comparaciones chlte tatamicnos $\left(D=322,85 ; X^{2}\right.$ ath $=$ 9.487 cona $=0.05 y g .1=4)$

El tatamicho con verde malaquila obluvo ha menor proporción de ov as mucrias por hongos, además se obscrió que el ácido acćlico a concenuraciones de 100 ppm y 300 ppm no presentó difcrencias signilicativas cn la proporción de ovas mucras por hongos. lo que hace a anbos matmicntos estadisticamente igunles. Finalmente se observó que la sal común, prescnió la mayor propor-

Tubla 1.

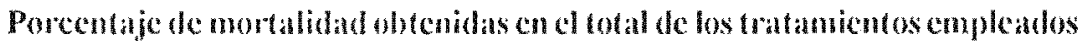

\begin{tabular}{|c|c|c|c|c|}
\hline Tratamicnto & Replica & $\begin{array}{l}\text { o/Mortalidad al } \\
\text { ovat con ojo }\end{array}$ & $\begin{array}{l}\text { M/oMorlalidad } \\
\text { atribuilla a bongos }\end{array}$ & $\begin{array}{l}\text { GoMortaliolan } \\
\text { Total a cclosion }\end{array}$ \\
\hline \multirow{4}{*}{$\begin{array}{c}\text { Verde } \\
\text { Malaquia } \\
2.5 \mathrm{ppm}\end{array}$} & 1 & 12.2 & 6.15 & 71.6 \\
\hline & 2 & 12.91 & 6.72 & 39.8 \\
\hline & 3 & 13.74 & 7.4 & 26.4 \\
\hline & Prom. & $12.95( \pm 0.77)$ & $6.76( \pm 0.62)$ & $45.9(( \pm 23.21)$ \\
\hline \multirow{4}{*}{$\begin{array}{c}\text { Sal } \\
\text { Comín } \\
20 \text { o/oo }\end{array}$} & 1 & 41.86 & 32.77 & 54.4 \\
\hline & 2 & 23.26 & 16.95 & 39.9 \\
\hline & 3 & $2+.71$ & 17.7 & +2.8 \\
\hline & Prom & $29.92( \pm 10.34)$ & $22.45( \pm 8.92)$ & $45.7(( \pm 7.67)$ \\
\hline \multirow{4}{*}{$\begin{array}{l}\text { Acido } \\
\text { Accilico } \\
100 \text { ppm }\end{array}$} & 1 & 21.86 & 16.74 & 71.8 \\
\hline & 2 & 20.93 & 17.35 & 54.3 \\
\hline & 3 & 20.19 & 15.67 & +2.2 \\
\hline & Prom & $20.90( \pm 0.83)$ & $16.50( \pm 0.85)$ & $63.0(0 \pm 14.88)$ \\
\hline \multirow{4}{*}{$\begin{array}{l}\text { Acido } \\
\text { Acćlico } \\
300 \text { ppm }\end{array}$} & 1 & 21.53 & 15.86 & 42.6 \\
\hline & 2 & 21.51 & 16.05 & 42.8 \\
\hline & 3 & 21.86 & $1+.75$ & +2.6 \\
\hline & Prom. & $21.6+( \pm 0.19)$ & $15.55( \pm 0,70)$ & $42.6((+0) .11)$ \\
\hline \multirow{4}{*}{$\begin{array}{l}\text { Acido } \\
\text { Acćlico } \\
500 \text { ppm }\end{array}$} & 1 & 14.38 & 9.02 & 30.0 \\
\hline & 2 & 18.89 & $13 .+3$ & 30.6 \\
\hline & 3 & 17.63 & 12.7 & 51.1 \\
\hline & Pron & $16.96( \pm 2.32)$ & $11.72( \pm 2.36)$ & $37.2(1 \pm 12.01)$ \\
\hline
\end{tabular}


ción de ovals mucrias por hongos del periodo (Fig, 1).

Con respecio a ha presencia de alevines con saco que prescntaron deformidad de la mandibula y/o curvatu-

Figura 1.

Porcentaje de mortalidad para los distintos tratumientos

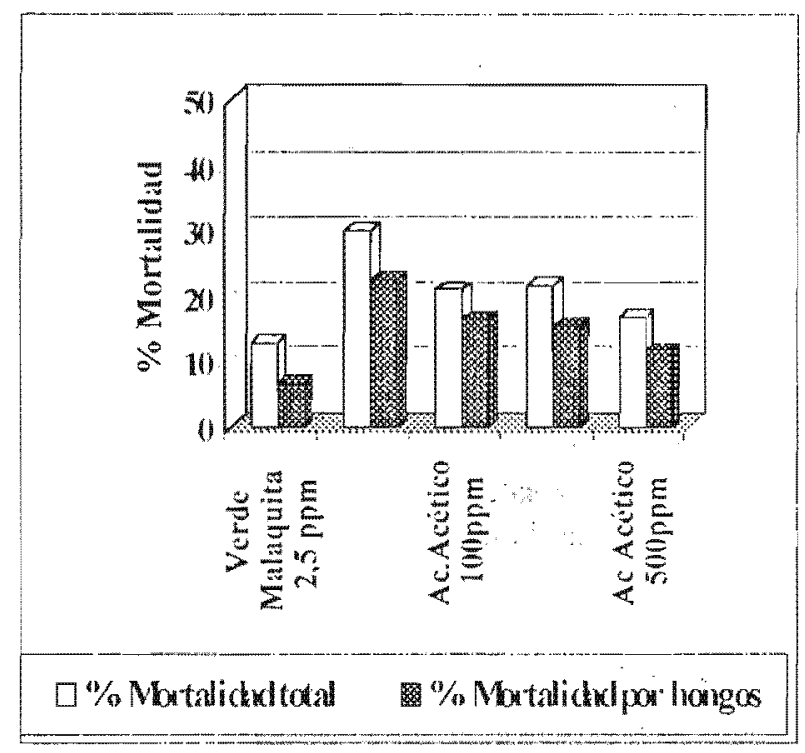

ra de la columa vertcbal. el verde malaquina presentó la mayor proporción de individuos deformes. con un promedio de $13.5 \%$. También fuc posible apreciar que los restantes tratamientos obtuvicron porcentaj]cs de deformidid muy similares. no cxisticndo cntre cllos diferencias de más de un $1 \%$. El tratamicnto que arrojó la menor cantidad de individuos deformes fucel áciclo acético a $500 \mathrm{ppm}$. con un porcentaje promedio de $6.17 \%$. La diferencia registrada por cl verde malaquia con el resto de los tmamicntos. supcra el $90 \%$. El análisis estadistico detcrminó que todas las funciones de culace propuestas permitian un ajuste satisfactorio de los modelos (D=27.95: $X_{\text {inthat }}^{2}=9.487 \mathrm{con}$ $\alpha=0.05$ y $\mathrm{g}, 1=4)$. El tratamicno de sill común y los de ácido acćtico a 100.300 y 500 ppm, no presentaron diferencias significativas en cuanto a deformidad $(\alpha=0,05)$ por lo que pucden ser considerados estadisticamente iguales entre sí. pero cstadísticamenic dierentes respecto al verde malaquita, que prescntó los mayores porcentijes de deformidad (Tabla 2).

Si se cralúan en conjumto has pérdidas por morlalidad atribuida a hongos y porcentaies de deformidad. sumando los hucros mucrios a los sobrevivientes que gencran alevincs defonmes, se puede estimar una pérdida por cada 1000 a aribuible a hongos de $20.3 \%$ para verde mala- quilay de $17.9 \%$ para ácido acético a 500 ppm. esta diferenm cha aungue far onble a ácido acético. no es esladísticamente significalliva al $5 \%$

Tablat 2.

Número de atevines con saco deforme por tratamicub en mucstra de 200 alevines

\begin{tabular}{|c|c|c|c|c|}
\hline Tratamiento & Rep. & $\begin{array}{l}\text { Defi. cn } \\
\text { Mamif- } \\
\text { bula }\end{array}$ & $\begin{array}{l}\text { Curata- } \\
\text { raten co- } \\
\text { lumana }\end{array}$ & $\begin{array}{l}N^{\circ} \text { Total } \\
\text { daformes }\end{array}$ \\
\hline \multirow{3}{*}{$\begin{array}{c}\text { Vorde } \\
\text { Malnquin } \\
2.5 p p m\end{array}$} & 1 & 17 & 11 & 28 \\
\hline & 2 & 16 & 11 & 27 \\
\hline & $\overline{3}$ & 10 & 16 & 26 \\
\hline \multirow{3}{*}{$\begin{array}{c}\text { Sill } \\
\text { Comin } \\
200 / 00\end{array}$} & 1 & 5 & 10 & 15 \\
\hline & 2 & 6 & 6 & 12 \\
\hline & 3 & 5 & 8 & 13 \\
\hline \multirow{3}{*}{$\begin{array}{l}\text { Acido } \\
\text { Acćlico } \\
100 \text { ppm }\end{array}$} & 1 & 5 & 8 & 13 \\
\hline & 2 & 3 & 12 & 15 \\
\hline & 3 & 8 & 6 & $1+$ \\
\hline \multirow{3}{*}{$\begin{array}{l}\text { Acido } \\
\text { Accilico } \\
300 \text { ppm }\end{array}$} & 1 & 7 & 7 & 14 \\
\hline & 2 & 6 & 9 & 15 \\
\hline & 3 & 9 & 4 & 13 \\
\hline \multirow{3}{*}{$\begin{array}{l}\text { Acido } \\
\text { Acćlico } \\
500 \text { ppm }\end{array}$} & 1 & 9 & 2 & II \\
\hline & 2 & 10 & 4 & 14 \\
\hline & 3 & 5 & 7 & 12 \\
\hline
\end{tabular}

\section{DISCUSION}

El cmpleo del verde malaquifa para minimizar la infección fungica de oras cn las pisciculturas fuc uno de los mélodo más utilizados a mivel mundial, hasta que se comprobó su poder lonlogénico (Merer \& Jorgensen. 1984; Schwaiger ef at. 1905). Además. csie coloranc conlamina cl candal de salida de estos centros de cultivo sicndo poicncialmone tóvico para la salud do los aminalcs (Bailey \& Jefrey 198\%: Marking ef al., 1994). Debido a cstos cfectos el US Fish and Wildifo Service y la Investigational New Animal Drug (INAD) prohibicron d uso del verde malaquita para el lramianto de hucros cn cl año [99].

En Chilc no cxisle una clan regulación sobre el uso de substancias inhibidoras del crecimicnto füngico $\mathrm{cn}$ estas fictorias y an se signe cmpleando ol verde de maliquita.

El cfeclo de los tratamientos en la deformidad de alcrines con saco, indica que al complear una dosis de ver- 
de malaquita de $2.5 \mathrm{ppm}$. a proporción de deformes que se obluro $(13,5 \%)$. cs mayor que co los restames tatamicntos, no obstante cntre cllos la diferencia cs minima. al canzando valores entre $6 \%$ y $7 \%$. Esto confirma los resullados obicnidos por Edgell er al. (1993) para salmón Colio (Oncorlynchus kisuldh) y Chinook (Oncorhynelus tshanytscha), los cuales sosticnen que ol verle malaguita a I ppm ticne alta incidencia cn la deformidad de alcrincs con saco $(16 \%)$. superando a tratamientos con solucioncs salinas $(9.3 \%$ y hucros sin thatar $(10.3 \%)$. Las diferencias en los porcentajes de deformidad registrados en muestma investigación y la de cstos illtimos antores. podrian deberse a los pirametros de deformidad cskiblecidos: Edgell et al. (1993). se basan en deformidades estructurales no especificadas y problcmas asociados a congulación de la yemal y saco vitclino. micntras que nuestros resultados se bilsan solo cn deformidades cstruchurales (curvalum de columma y defomidad de mandibula),

Las semejanzas observada cutre los diferentes talamicntos de ácido acćlico y clonuro de sodio a $20 \%$ podrian deberse a que estos clenentos no inciden en la deformidad de alevincs con saco y por lo tanto. podrim asociarse a porcentajes nomales de deformidad pan individuos gue en eshado de ova verde no hayan sido sometidos a ningin fralamicnto.

El verde malacuiti confirma cn nuestros resultados lo sosicnido por Mcycr \& Jorgensen (1984) $\mathrm{cn}$ relación a que el uso de este clemento provoca cfeclos mutagénicos y teralogénicos que pucden ser aprecindos por la presencia de alevines con mormalidades $\mathrm{Cn}$ COlumma cabcza alcas y cola. Autores como. Alderman (1991). Schwaiger et al. (1945) schatun que las deformidides se cleben a que este elemento actua a nivel cromosónico.

Las mayores mortalidades totales atribuidas a hongos. ocurricron cn los tralamionos con clontro de sodio. no obstante la sal común. sal de mary mezclas de clonuro de sodio y clomro de calcio se han sugerido para controlar hongos durante la clapa de incubación. además el cloruro de sodio se ha utilizdo ampliamente como agcnte terapéutico y para reducir el estrós de los peces en cintiverio (Schroict at al. 1996).

Taylor \& Bailoy (1979) señalan que introducichdo los hucios de salmón rosado (O. gorbuschu) cn agual de mar clumic 2 a 3 horas diarias se pucde controlar cfectivancnte Saproleznia dictima. Marking el al. . (1994) cncontaron, en huevos de salmón que soluciones salinas de $20 \%$ son lan efeclivas on el control de hongos como l ppm de verde malaquita, similares resullados fucron reportados por Edgell et al. (1993) quicnes al tratar hucros de salmon Chinook (Oncorhynchus tshanytscha). con cloruro de sodio a concentraciones de $15 \% .17 .5 \%$ y. $20 \%$ por 60 minutos y tres veces por semima. regist raron morta- lidades de $20.7 \%$. $17.1 \%$ y $10.2 \%$ respectivamene, csic ullimó porcentajc fue similar al que obturieron con verde malaquila. que presento mortalidades de $8.8 \%$. En nucstra incesligación. la montalidad observada al $20 \%$ de clonuro de sodio, fue de $29.95 \%$ a pesar de contar con un tiempo de duación similar c igual periodicidad en la aplicación del matamicnto: a diferencia culre los resultados podrian deberse a las condiciones cxperimentales.

La clicacia del ácido acćico se asocia con su acción anthicrobiana que Femena (1993). considera similar a la de olros ácidos usidos en la preservación de alimentos. ya que csta aumenta al disminuir $\mathrm{cl} \mathrm{pH}$. Adcmás scinala que la propicdad de imhibir la reproducción. cl crecimiento y por lanto la aclividad de los microorginismos. se debe a que este clemenlo puede generar intererencias on las actividades cuzimáticas y on la mombrana colular. Schmidt-Hcbbel (1979). sostiene que la molćcula no disociada del ácido penetra la membrana celular de los microorganismos " se disocia dentro de has células. inerementundo la acidez y produciendo niveles tóxicos. De los tralamientos con ácido acético cmpleat dos. el que prescuto mejores resultudos, fte a $500 \mathrm{ppm}$. Marking et.al. (1994). Scrialan que cl ácido acético a 300 ppm inhibe el crecimicnto de hongos, a 400 ppm es efectiro para controlar suaparición y sobre 1000 ppm son tóxicos para los hucros, no obstante, estos autores no recomiendam su uso como fungicida por la acidificación del medio.

Basindonos en Fennema (1993) y Schmidt-Hebbel (1979). consideramos que csla reducción del pH cxplicaria la efectividad del ácido acético a 500 ppm: cn cambio las menores. resultaron ser cstadisticamente iguales. Nuesthis diferencins en los resuliados. pueden deberse a las condiciones experimentales en que se realizaron los estudios: la investigación de Marking et al. (199+). se cfecuó a temperatura de $12^{\circ} \mathrm{C}$. cu batcas verticales de incubación y Mujos de agua constanles. inocukndo los huevos con hongos. En cambio en nuestro estudio, se utilizaron batteas honizonales. Mlujos de agua variables y temperaturas chitre $1^{\circ} \mathrm{Cy} 8^{\circ} \mathrm{C}$.

Es posible concluir que el ácido acćtico cn concentraciones de 500 ppm puede ser utilizado como sustituto al rede malaquita para controlar infecciones micólicals cl ovas.

\section{REFERENCIAS}

Nderman, 1). (1982). In vitro lesting of lisheries elemotherapeutams. Joumal of Fish Diketases 5:113-123

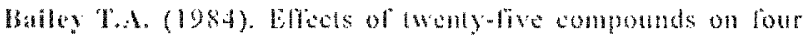

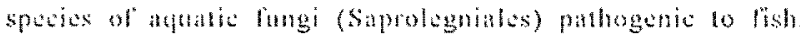
Aquatedhure 38:97-104

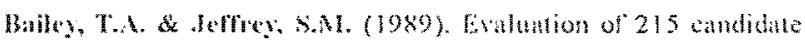




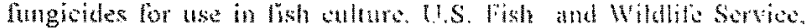
Investigntions in Fish Control 99

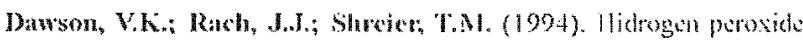

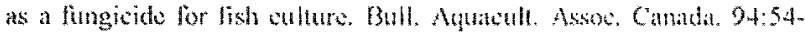
56

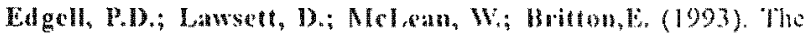
use sall solutions to comtrol fungus (Saprolegina) inlintutions on salmon eggs. Brogressive lish-evllurict, $55: 42 \times 48$

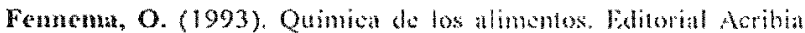
\$.A.Zaragoza.

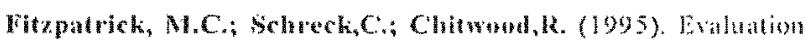
of tres candidate fungicides for trentmont of adult pring chinosk salmon. Progressive fish-cult:57:153-15

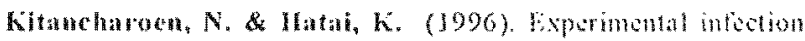
of Saprolegnia spp. In ranbow trout eggs. Fish-1 alhol. 31:49-50

Marking, L.L.; Rach, J.s.; Scherefe, T.M. (1994). Evaluation of

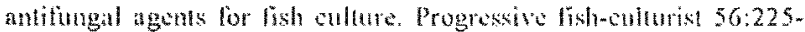
231

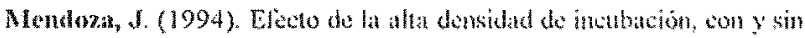

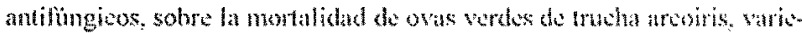

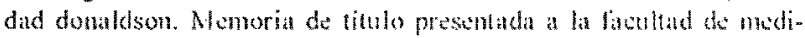

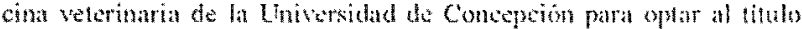
de Médico veferinato, Chillin, Chils.

Meger, F.P. \& Vrgensen, T.A. (1983). Termologion and other efteds of malachite green on development in rahbits and rainhow irout. Trans of the Amer. Iish. Soc. 112:818-824

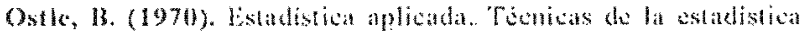

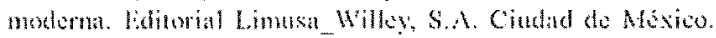

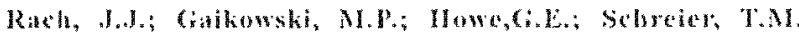
(1998). lisaluation of the loxicity and dhatey of hathen poroxido

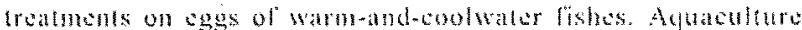
$165: 11-25$

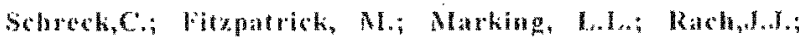

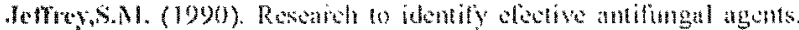

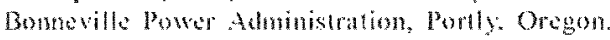

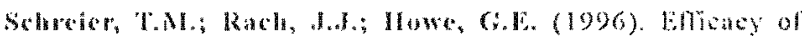

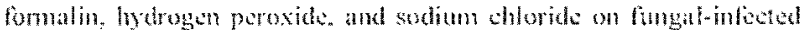

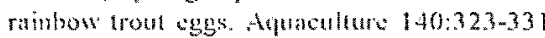

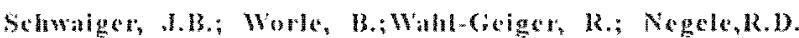

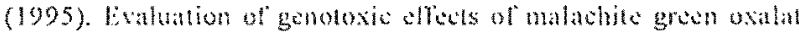

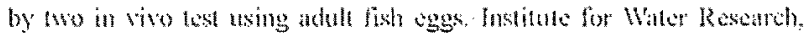
Divarian Staks.

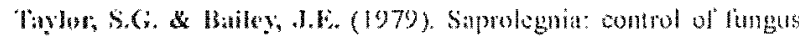
on incubation sags of pink salmon by frobment with seawater. progressibs hish-eulurist, $41: 181+183$

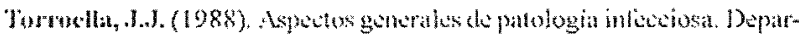

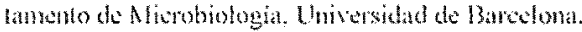

Millazenby, I. I. \& Raberts, Rol. (1992). Towards strategit the

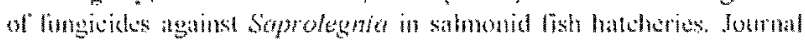
ol Wish Dikente 15:1-13 